\title{
The Role of Sample Collection and Scanning Electron Microscopy (SEM) in Contaminant Analysis in Hospital Pharmacies
}

\author{
Farrell Melnick, * Himansu Bhattacharjee,* Richard J. Lee, ${ }^{* *}$ \\ * R.J. Lee Group, Inc, $3505^{\text {th }}$ Ave., New York City, NY 10118 \\ ** R.J. Lee Group, Inc, 350 Hochberg Road, Monroeville, PA 15146
}

The US Pharmacoepia (USP) has issued new standards designed to regulate the preparation of sterile compounds in hospital pharmacies. The regulations, referred to as USP 797, mandate environmental monitoring in the work area to confirm that the pharmacy is within certain guidelines regarding particulate and microbial contamination [1]. The new standard is designed to reduce the possibility of contamination of pharmaceutical preparations. The standard, introduced on January 1, 2004, is enforceable by the U.S. Food and Drug Administration. It has since been adopted by some pharmacy and regulatory bodies, especially some state boards of pharmacy and the Joint Commission on Accreditation of Health Care Organizations (JCAHO) [2]. Although the standard does not mandate a specific form of sampling, general references list the following alternatives: Microbial sampling including passive air sampling with agar impregnated settling plates, and/or active air sampling with either centrifugal samplers, impaction samplers or liquid impingement; surface sampling with either contact plates, swabs or agar paddles; and particulate sampling including electronic particle counter and/or aerosol challenge test [3].

We have developed an approach to ascertain microbial and particulate contamination based upon a combination of real time reading for particulate, Andersen impactor with selectively chosen plates for microbes in air, and use of a wiping process with scanning electron microscopy analysis for total particulate and microbes on a work surface.

The latter is performed by wiping laminar flow or other clean room work surfaces with sterilized cleanroom wipers. This active method collects particles, microbes and other debris embedded on the work surfaces. The current clean room standard technique, ASTM E-2090, involves immersing the wiper in a standard low-surface-tension cleaning liquid such as a surfactant/water solution and then subjecting to mechanical agitation in that liquid [4]. The entire amount of the liquid containing the particles, fibers and other debris in suspension is filtered through $0.2 \mathrm{um}$ microporous membrane filter. The collected debris is inspected by Optical (OM) and Scanning Electron Microscopy (SEM) after depositing gold coats.

The major advantage of the method includes seeing actual particulate shapes and sizes spread over the filter. The use of OM and SEM as viewing and enumeration tools provides a direct and precise measurement of the quantity and type of contamination including microbes. Although there are no specific standards for surface contamination in the pharmacy, the usefulness of the SEM lies in its ability to provide clear, high resolution images that can often differentiate between ordinary particulates and microbes. These results are critical in providing the hospital pharmacist with information regarding the nature and possible sources of contamination. The photomicrographs shown below are typical examples from surfaces of a sterile preparation area using wiping sampling and scanning electron microscopy analysis. 
References

[1] US Pharmacopeial Convention, Inc. US Pharmacopeia 27. Chapter 797, Pharmaceutical - Sterile Preparations. Rockville, MD: The United States Pharmacopeial Convention, Inc.; 2004: 2350-2370

[2] Drug Standards, Proposed Revisions to USP Chapter 797; Pharmaceutical Compounding Sterile Preparations. The United States Pharmacopeial Convention, Inc. USP web site, 1-06-2005

[3] E. S. Kastango, Environmental Monitoring. Chapter 18, Compounding Sterile Preparations, $2^{\text {nd }}$ Edition; E. Clyde Buchanan and Philip J. Schneider, American Society of Health-System Pharmacists, 2005.

[4] ASTM E-2090-00, Standard Test Method for Size-Differentiated Counting of Particles and Fibers Released from Cleanroom Wipers using Optical and Scanning Electron Microscopy, July, 2000.

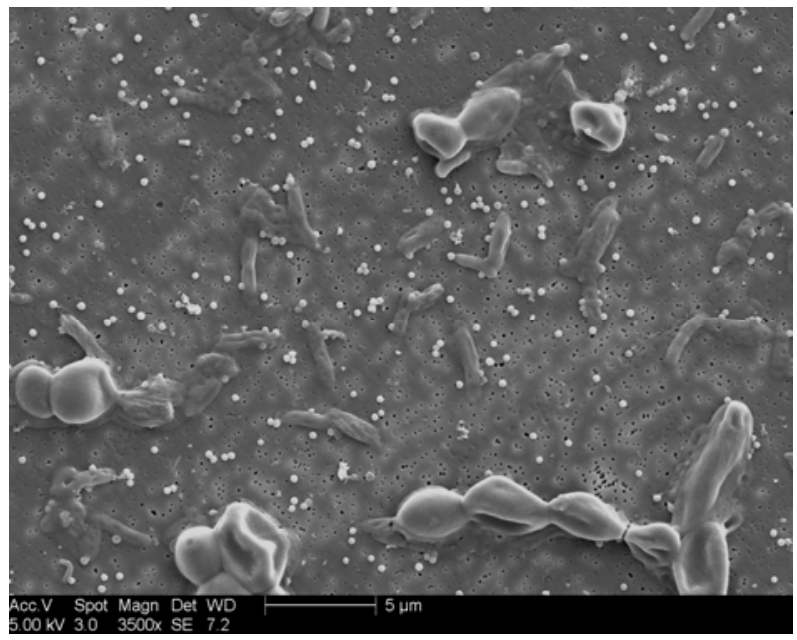

FIG. 1. SEM Photomicrograph showing surface bacterial contamination at 3500X.

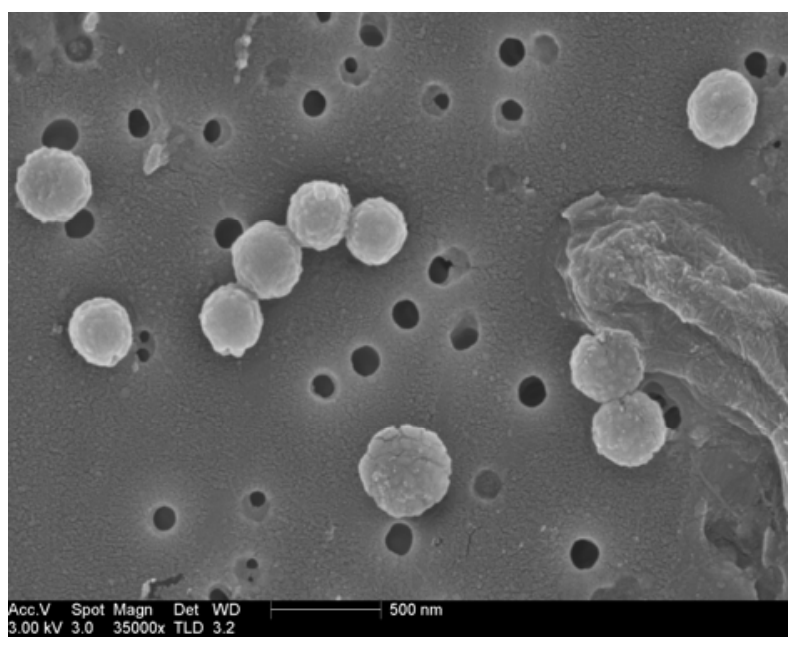

FIG. 2. SEM Photomicrograph showing bacterial contamination at $35000 \mathrm{X}$. 